\title{
Using the score method to construct asymmetric confidence intervals: An SAS program for content validation in scale development
}

\author{
JEFFREY M. MILLER \\ University of Florida, Gainesville, Florida \\ and \\ RANDALL D. PENFIELD \\ University of Miami, Coral Gables, Florida
}

\begin{abstract}
Expert review sessions are often conducted to determine the content validity of scale items. The accurate quantification of content validity is usually limited by a relatively small number of experts as well as by a small number of rating categories. These factors, combined with the bounded and discrete nature of rating scale categories, hinder use of traditional methods for computing standard errors and confidence intervals. Using an application of the score method, researchers can construct an asymmetric interval that is better suited for these situations. SAS code is provided to automate the computations, and a discussion of two methods for using the obtained results for content validation decisionmaking follows.
\end{abstract}

Crocker (2003), in her presidential address to the National Council on Measurement in Education, stressed the importance of improving quantification procedures for content validation in test construction. One widely used procedure in which a group of experts, usually fewer than 10, rates the relevance of the content of a scale item (American Educational Research Association et al., 1999; Crocker, 2003; Crocker, Miller, \& Franks, 1989; Geisinger, 1992; Shepard, 1993; Sireci, 1998) illustrates this need. Typically, the mean of the scale rating is used as a measure of endorsement for the content validity of the item. If the rating scale is bounded from 1 to 5 , where 5 represents strongly agree, a mean rating of 4.7 would be interpreted as relatively strong agreement for the content validity of the item in question. This mean rating, however, does not provide information regarding its accuracy as an estimate of the true population mean rating.

One procedure for determining the accuracy of sample mean ratings is to construct a Wald confidence interval. In this procedure, which is taught in most introductory statistics courses, the confidence interval has upper and lower bounds defined by

$$
\bar{X} \pm t_{d f}\left(\frac{s}{\sqrt{n}}\right) .
$$

Hence, the Wald interval uses the mean rating, the critical $t$ statistic, and the standard error of the mean rating.

Correspondence concerning this article should be addressed to J. M. Miller, College of Education, University of Florida, P.O. Box 117047, Gainesville, FL 32622-7047 (e-mail: millerjm@ufl.edu).
Unfortunately, the Wald interval is inappropriate when one is constructing confidence intervals for rating scale means: First, the number of raters is usually too small to accommodate the assumption of normality, and second, the Wald interval can produce limits above and below the actual limits of the scale. For example, if the scale has a minimum value of 1 representing strongly disagree, the Wald interval could produce an uninterpretable lower limit of .20. Third, there is no reason to suspect that the item rating distribution is symmetric. When all raters strongly agree that the item content is representative of the intended content domain, a negatively skewed distribution is expected.

One solution to this predicament is to develop a confidence interval that is (1) not limited by the assumption of population normality and (2) less affected by the bounded nature of rating scale items. Such an interval would be based on the properties of the distribution of the item itself regardless of symmetry. Wilson (1927) presented such an interval based on the mean of a dichotomous variable.

Penfield (2003) generalized Wilson's score method to the mean of a rating scale item. The results of the simulations suggest that the score method outperforms the traditional Wald interval, especially when the number of response options is small (e.g., three) and when the sample size is small (e.g., five to seven). The small sample conditions prompted the application of the score method to constructing confidence intervals about mean rater endorsements in the content validation process. Penfield and Miller (2004) described an application of the score method to content validation as well as to the computational procedures. 
Computational complexity limits the effectiveness of other indexes for content validation (e.g., Aiken, 1980, 1985). Although the score confidence interval for the rating scale mean is an improvement over alternative procedures, the calculations may still discourage the applied use of the interval. To this end, SAS code has been constructed to facilitate calculation of the interval.

\section{METHOD}

\section{Statistical Calculation}

The first step in the score method for constructing the confidence interval is to obtain the value for $n$ (the number of experts providing ratings), $k$ (the number of response options on the Likert scale), and $z$ (the standard normal deviate corresponding to the desired confidence intervali.e., \pm 1.96$)$. The second step requires computing the mean item rating across all $n$ raters. Third, $\pi_{\mathrm{L}}$ and $\pi_{\mathrm{U}}$ are calculated through the following two equations:

$$
\pi_{\mathrm{L}}=\frac{2 p n k+z^{2}-z \sqrt{4 n k p(1-p)+z^{2}}}{2\left(n k+z^{2}\right)}
$$

and

$$
\pi_{\mathrm{U}}=\frac{2 p n k+z^{2}+z \sqrt{4 n k p(1-p)+z^{2}}}{2\left(n k+z^{2}\right)},
$$

where

$$
p=\frac{\sum_{i=1}^{n} R_{i}}{n k}
$$

if the Likert scale ranges from 0 to $k$, or where

$$
p=\frac{\sum_{i=1}^{n} R_{i}}{n k}-\frac{1}{k}
$$

if the Likert scale ranges from 1 to $k$. Finally, the confidence interval is calculated by using the following two equations:

$$
\bar{R}-z \sqrt{\frac{k \pi_{\mathrm{L}}\left(1-\pi_{\mathrm{L}}\right)}{n}}
$$

for the lower limit, and

$$
\bar{R}+z \sqrt{\frac{k \pi_{\mathrm{U}}\left(1-\pi_{\mathrm{U}}\right)}{n}}
$$

for the upper limit.

\section{Using SAS to Calculate the Interval}

SAS is a statistical programming language commonly used in psychology, education, and industrial research. The SAS code for the score method of calculating the confidence interval for the mean of a rating scale item is displayed below. This code and the SPSS code are also available through the journal archives.

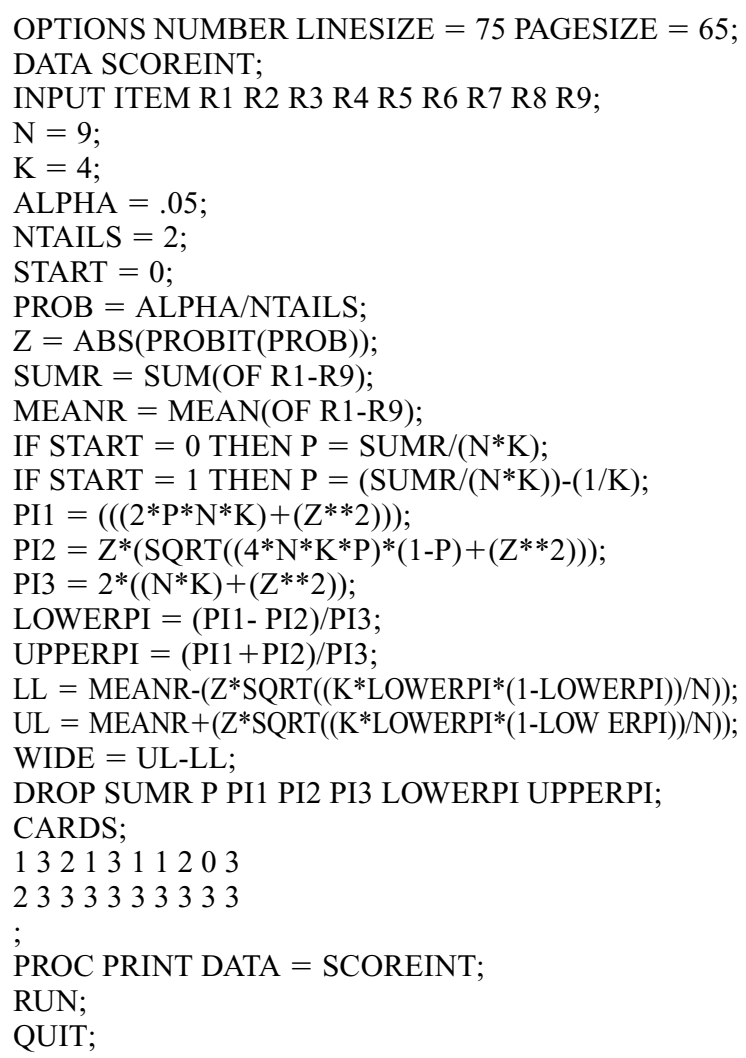

The third line declares a variable for each rater (e.g., R1, R2, R3). This can be adjusted to accommodate the number of raters in the content validation procedure, but the following line would also need to be adjusted. For this example, there were nine raters. Hence, variables were declared for R1 through R9, and the following line was declared $N=9$. The fifth line declares the number of rating scale categories. For this example, the rating scale was from 0 to 3 ; that is, $k=4$. The following lines declare the desired Type I error rate (e.g., .05, .01), the number of tails associated with the confidence interval (i.e., 1 or 2), and the starting value, which is the lowest possible category response option (i.e., usually 0 or 1).

The program has a built-in adjustment to accommodate for scales that begin with either 0 or 1 . The raw data is entered after the CARDS command. The first entry is the item number; thus, this program permits the analysis of ratings for all items in one execution. There are two items being rated in this example. Following this index are the ratings given by the raters - nine ratings for this example.

The output provided by SAS for this example is shown in Table 1.

As a method of double-checking for accuracy of input, SAS restates, after the observation number, the item number, the nine ratings, the sample size $(\mathrm{N})$, the number of rating scale categories $(\mathrm{K})$, the Type I error rate (ALPHA), the number of tails (NTAILS), and the lowest possible rating (START). This is followed by the Type I 
Table 1

\begin{tabular}{|c|c|c|c|c|c|c|c|c|c|c|c|c|c|c|c|}
\hline Obs & item & $\mathrm{R} 1$ & $\mathrm{R} 2$ & R3 & R4 & R5 & R6 & R7 & $\mathrm{R} 8$ & R9 & $\mathrm{N}$ & $\mathrm{K}$ & Alpha & Ntails & Start \\
\hline 2 & 1 & 3 & 2 & 1 & 3 & 1 & 1 & 2 & 0 & 3 & 9 & 4 & 0.05 & 2 & 0 \\
\hline 2 & 2 & 3 & 3 & 3 & 3 & 3 & 3 & 3 & 3 & 3 & 9 & 4 & 0.05 & 2 & 0 \\
\hline Obs & \multicolumn{3}{|c|}{ Prob } & \multicolumn{2}{|l|}{ Z } & \multicolumn{3}{|c|}{ Meanr } & \multicolumn{2}{|c|}{ LL } & \multicolumn{3}{|c|}{ UL } & \multicolumn{2}{|l|}{ Wide } \\
\hline 1 & \multicolumn{2}{|c|}{0.025} & \multicolumn{3}{|c|}{1.95996} & \multicolumn{3}{|c|}{1.77778} & \multicolumn{2}{|c|}{1.18165} & \multicolumn{3}{|c|}{2.37390} & \multicolumn{2}{|l|}{1.19225} \\
\hline 2 & \multicolumn{2}{|c|}{0.025} & \multicolumn{3}{|c|}{1.95996} & \multicolumn{2}{|c|}{3.00000} & & \multicolumn{2}{|c|}{2.35718} & \multicolumn{3}{|c|}{3.64282} & 1.28564 & \\
\hline
\end{tabular}

error rate adjusted for the number of tails (PROB) and the provided critical $z$ statistic associated with the number of tails and Type I error rate.

The output then provides the mean item rating. For the first item, the mean rounds to 1.78 , indicating relatively low endorsement for this particular item. The output that follows then supports this notion with a confidence interval ranging from $\mathrm{LL}=1.18$ to $\mathrm{UL}=2.38$ with a width of WIDE $=1.19$. It is unlikely that the low mean rating is a result of one dissident rater. The mean rating for the second item is 3.00; however, the confidence interval for this item ranges from $\mathrm{LL}=2.36$ to $\mathrm{UL}=3.6$ with a width of WIDE $=1.29$.

\section{DISCUSSION}

For the first item, the score interval $(1.18,2.38)$ has a width of 1.19. In comparison, the traditional Wald interval for this item $(0.94,2.62)$ has a width of 1.68 . Hence, the narrower score interval results are consistent with previous research. Furthermore, because the score interval does not rely on normality assumptions, the limits should be more indicative of the actual distribution for the responses to this item than would limits derived using other procedures. For the second item, a traditional Wald interval is not possible because all responses were identical. In this situation, the score interval provides information regarding potential response limits.

The efficient automation of the score method for constructing an asymmetric confidence interval about the mean item rating promotes two applications. First, the content experts may determine a priori that they will accept only items that have a confidence interval width of less than 1.2. In this case, the first item would be retained, but the second item would be dropped or modified. Second, the content experts may determine a priori to accept only items that have a lower limit of relatively strong endorsement. In this example, a rating of 0 represents strong disagreement, and a rating of 3 represents strong agreement. If a rating of 2 were determined to be relatively strong agreement, then, under the predetermined conditions, the first item would be dropped and the second item would be retained. Thus, the two decision criteria could yield different results regarding the fate of the item. Potentially, the two criteria could be combined for an even more valid approach to content validation. Regardless, either approach is more valid than a decision based on the mean alone. Furthermore, as demonstrated by Penfield (2003), the traditional Wald confidence interval is not only inappropriate but is potentially inaccurate when sample size and the number of rating categories are small. The application of this program to expert agreement of content in new scales presents a great improvement to technical reports that typically validate content as "the experts agreed" or that report a potentially misleading rating of mean endorsement.

\section{REFERENCES}

AIKEN, L. R. (1980). Content validity and reliability of single items or questionnaires. Educational \& Psychological Measurement, 40, 955959.

AIKEN, L. R. (1985). Three coefficients for analyzing the reliability and validity of ratings. Educational \& Psychological Measurement, 45, 131-142.

American Educational Research Association, American Psychological Association, \& National Council on Measurement in Education (1999). Standards for educational and psychological tests. Washington, DC: American Educational Research Association.

CROCKER, L. (2003). Teaching for the test: Validity, fairness, and moral action. Educational Measurement: Issues \& Practice, 22, 5-11.

Crocker, L., Miller, M. D., \& Franks, E. A. (1989). Quantitative methods for assessing the fit between test and curriculum. Applied Measurement in Education, 2, 179-194.

GeIsIngER, K. F. (1992). Fairness and selected psychometric issues in the psychological testing of Hispanics. In K. F. Geisinger (Ed.), The psychological testing of Hispanics (pp. 17-42). Washington, DC: American Psychological Association.

Penfield, R. D. (2003). A score method of constructing asymmetric confidence intervals for the mean of a rating scale item. Psychological Methods, 8, 149-163.

Penfield, R. D., \& Miller, J. M. (2004). Improving content validation studies using an asymmetric confidence interval for the mean of expert ratings. Applied Measurement in Education, 17, 359-370.

SHEPARD, L. A. (1993). Evaluating test validity. In L. Darling-Hammond (Ed.), Review of research in education (19th ed., pp. 405-450). Washington, DC: American Educational Research Association.

SIRECI, S. G. (1998). The construct of content validity. Social Indicators Research, 45, 83-117.

Wilson, E. B. (1927). Probable inference, the law of succession, and statistical inference. Journal of the American Statistical Association, 22, 209-212.

(Manuscript received March 12, 2004; revision accepted for publication August 13, 2004.) 